\title{
LA PLAZA Y EL USHNU DE HUALFÍN INKA: INTERPRETACIONES ACERCA DE SU FUNCIÓN EN LA ARQUITECTURA PÚBLICA DEL PERÍODO INKAICO
}

\section{THE PLAZA AND THE USHNU OF HUALFIN INKA: INTERPRETING THEIR FUNCTION IN THE PUBLIC ARCHITECTURE OF THE INKA PERIOD}

\section{JULIETA LYNCH*}

\begin{abstract}
A través del estudio realizado en dos estructuras sumamente importantes al momento de interpretar las estrategias de control inkaicas, la plaza y el ushnu, reflexionamos acerca de la dinámica de expansión en la región surandina y sus implicancias sobre las poblaciones locales del centro de la Provincia de Catamarca, Argentina. El presente artículo intenta aportar datos valiosos a la discusión de este tema, que se enmarca en una política de expansión del Tawantinsuyu y que no solo incluye el poder de la coerción, sino también numerosas formas de negociación e inclusión de las comunidades locales a la estructura estatal.

Palabras clave: plaza, ushnu, arquitectura, período inkaico, Noroeste Argentino
\end{abstract}

Through the study of the plaza and the ushnu-two major structures of central importance for interpreting Inka control strategies-we reflect on the dynamic of Inka expansion in the South-Andean region and its consequences for local communities in the Province of Catamarca, Argentina. This article attempts to provide valuable information on this topic, framed in the context of Tawantinsuyu's expansion policy, which included not only coercive power but many forms of negotiation and the incorporation of local communities into the state structure.

Key words: plaza, ushnu, architecture, Inka period, Northwest Argentina

\section{INTRODUCCIÓN}

El Imperio Inka, o Tawantinsuyu, fue quizás el sistema político de mayor extensión en América en épocas prehispánicas. El mismo se extendió desde Ecuador hasta Chile y Argentina a lo largo de la cordillera de los Andes, abarcando aproximadamente $4000 \mathrm{~km}$ de extensión (fig. 1).

Hacia 1532, cuando se produce la conquista española, el Imperio dominaba diferentes grupos étnicos, con distintas lenguas, costumbres, que se ubicaban a lo largo de Ecuador, Perú, Bolivia, Chile y Noroeste de Argentina, siendo su centro la ciudad de Cusco, Perú. Esta se encontraba bajo el dominio del último inka Pachacutec Inka Yupanqui.

En este breve período, de no más de cien años, los inkas extendieron sus fronteras y dominación sociopolítica sobre los reinos y etnias del altiplano, la sierra, los valles y la costa pacífica, ampliando sus límites desde el Cusco a lo largo de la cordillera de los Andes y cubriendo aproximadamente una superficie de un millón de kilómetros cuadrados. Se estima que en el momento de máxima expansión había una población total aproximada de seis a doce millones de habitantes, todos bajo un estricto sistema de tributos y burocracia creados por los inkas, que utilizaron e impusieron la lengua quechua en casi todo el ámbito de su dominio, conformando uno

\footnotetext{
* Julieta Lynch, Departamento Científico de Arqueología, Facultad de Ciencias Naturales y Museo (FCNyM, UNLP, Conicet). Museo de La Plata, Paseo del Bosque s/n, CP 1900, La Plata, Argentina, email: julietalynch@yahoo.es
} 


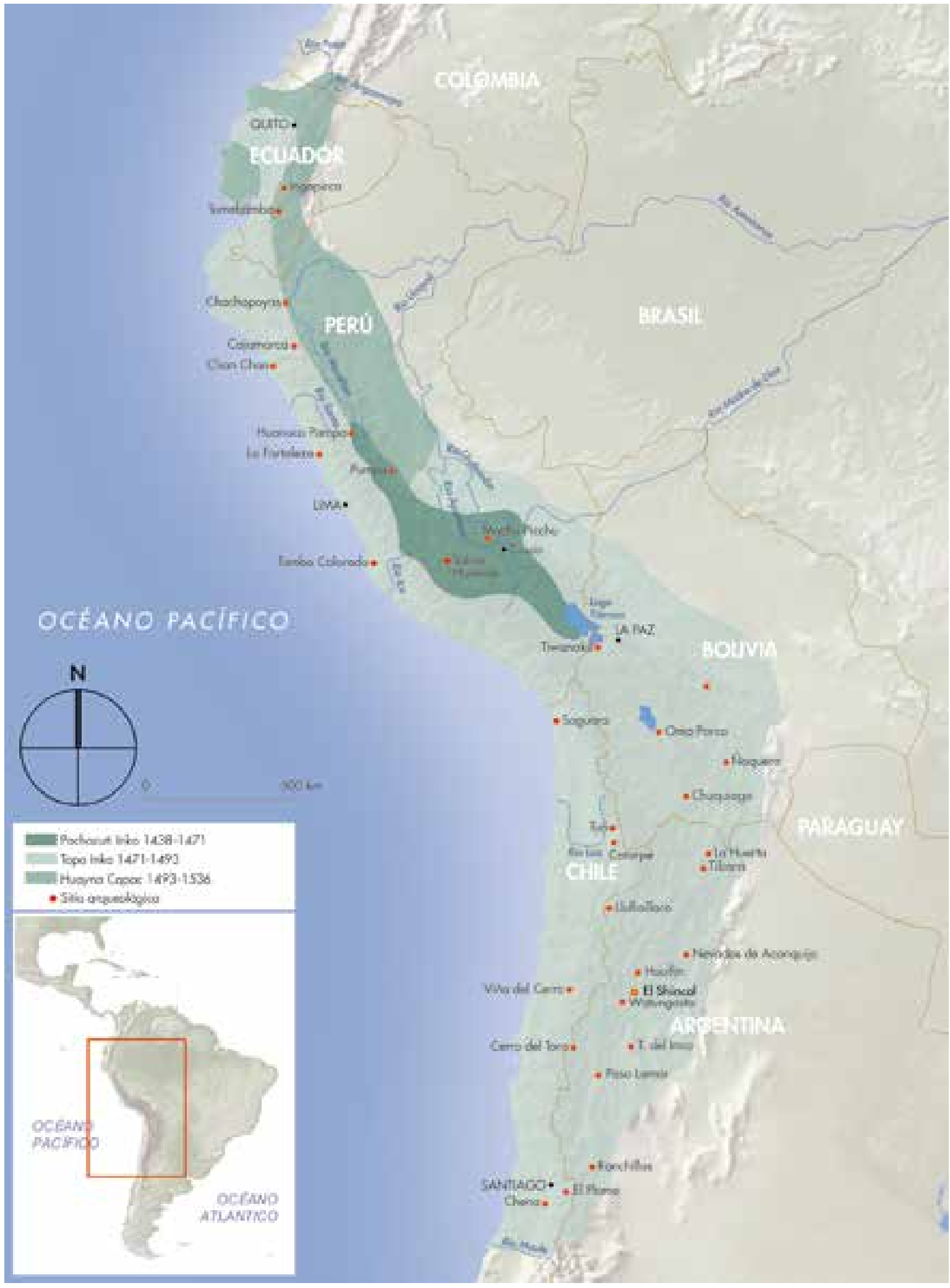

Figura 1. Expansión del Imperio Inka en América del Sur. Figure 1. Expansion of the Inka Empire in South America. 
de los estados más extensos y poblados de la América prehispánica (D’Altroy 1987; Stehberg 1995).

El sector meridional del imperio, denominado en quechua Kollasuyu, incluía el territorio actual del Noroeste Argentino (NOA), norte de Chile y sur de Bolivia (fig. 2), y se presume fue ocupado por los inkas en el siglo $\mathrm{xV}$, posiblemente en la segunda mitad. ${ }^{1}$ Los cambios producidos fueron complejos y debieron evaluarse región por región, dado que las estrategias implementadas por el Tawantinsuyu fueron múltiples y variables. Quizás lo más importante fue la conformación de un espacio social panandino, en el que muchos elementos preinkaicos fueron incorporados a la estructura mayor. Aspectos relacionados al culto a los antepasados, por ejemplo, cobraron una importancia que trasciende el espacio comunal local (Nielsen 2006).

El estudio de la presencia inkaica tiene raíces muy profundas en la construcción de la historia andina.
Desde las primeras crónicas hasta las investigaciones actuales, los estudios históricos y arqueológicos se han complementado eficazmente en la comprensión del orden sociopolítico y las características económicas, religiosas e ideológicas de este vasto imperio. Por su parte, las interpretaciones acerca de las motivaciones de la expansión y las características de la instalación del estado centroandino en estas regiones han mostrado un notable proceso de transformación, incluyendo desde explicaciones económico-funcionales hasta aquellas políticas e ideológicas.

Para el Noroeste Argentino se han propuesto diversas explicaciones en lo que se refiere al proceso de consolidación de este imperio, entre ellas podemos mencionar:

- La instalación de centros estatales a lo largo del camino principal y vías secundarias (Raffino 1981; Hyslop 1984, 1990; Vitry 2000).

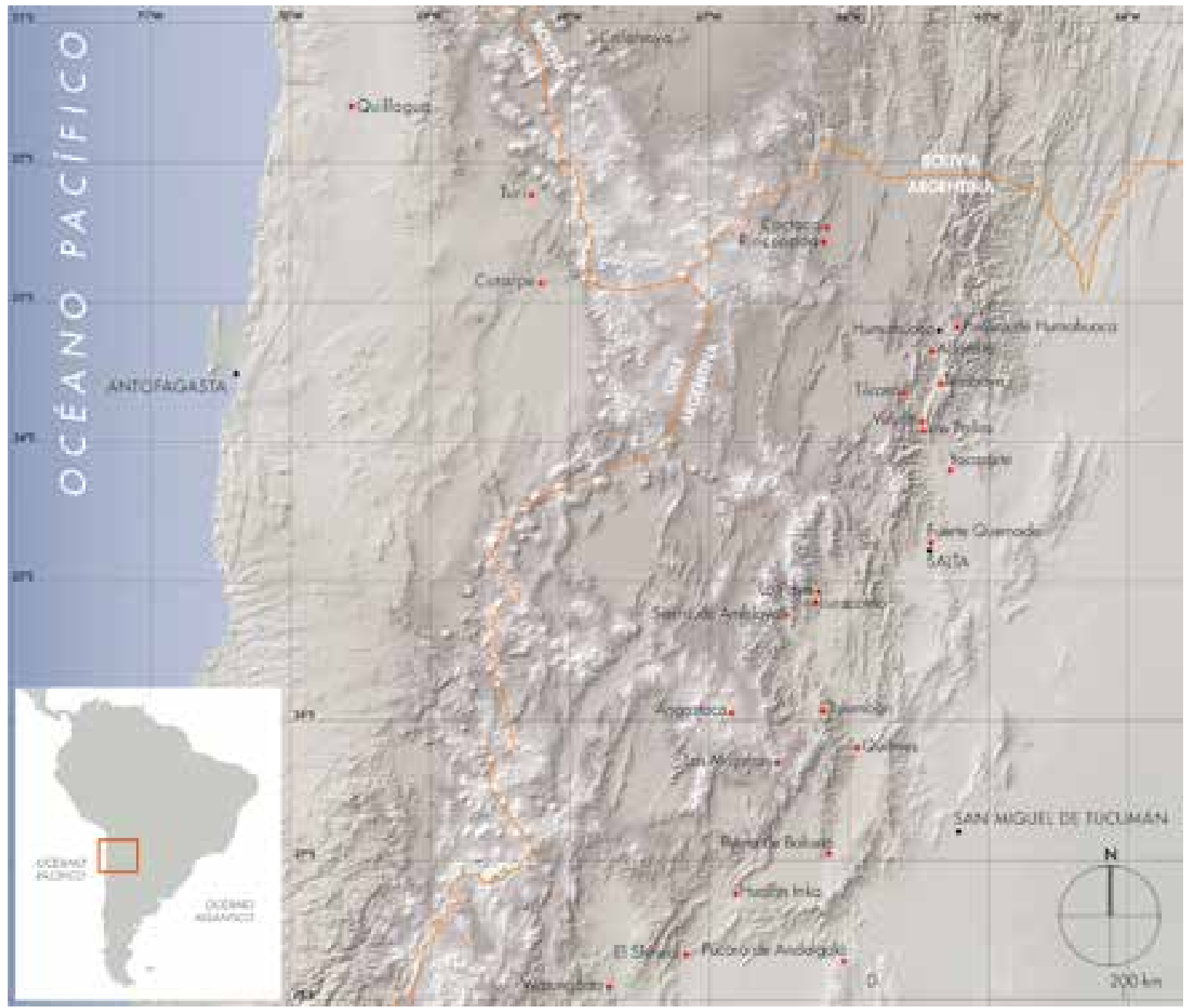

Figura 2. Localización de los sitios inkas en el Kollasuyu (basado en Williams 2004).

Figure 2. Location of Inka sites in Kollasuyu (based upon Williams 2004). 
- La instalación de fortalezas a lo largo de sus fronteras y de la red vial para mantener la seguridad (Williams 2004: 210).

- La intensificación de la producción agropastoril a partir del desarrollo de recursos separados de los de las sociedades nativas.

- La intensificación de la producción minera y artesanal (Raffino 1981).

- El reclamo del paisaje sagrado a través, por ejemplo, de la construcción de santuarios de altura ubicados a más de 5000 msnm (Schobinger 1966, 1971; Reinhard 1985; Ceruti 1997; D'altroy et al. 1998).

Investigaciones recientes revelan la complejidad de este fenómeno, en el que numerosos elementos paisajísticos se articularon dentro de la cosmovisión del Tawantinsuyu (Acuto 1999; Hyslop 1990; Inomata \& Coben 2006; Nielsen \& Walkers 1999; Willliams 2004).

El presente trabajo da cuenta de dichas estrategias ideológicas y simbólicas, en las cuales las estructuras arquitectónicas públicas -como las plazas y los denominados ushnus o plataformas artificiales de índole ceremonial-, constituyen un punto central en la profundización del mencionado fenómeno. Se presenta aquí el análisis de estas estructuras realizado en el sitio Hualfín Inka, en el valle de Hualfín, Catamarca, Argentina, a fin de profundizar estas diversas estrategias implementadas por el Inka al momento de anexar nuevos territorios.

Es necesario además resaltar el término visibilidad o campo visual que un ser humano puede obtener del entorno desde un sitio particular. La visibilidad implica tanto el emplazamiento como las estructuras construidas y las características del paisaje, que condicionan hacia y hasta dónde se puede ver desde un sitio (Borgstede \& Mathieu 2007). En nuestro caso, interesan sobre todo las líneas de visibilidad respecto de los espacios transitables dentro del sitio, y a determinados puntos geográficos relevantes en relación con la movilidad dentro del valle mismo y desde afuera hacia dentro del territorio.

Otro punto importante que se tuvo en cuenta en este trabajo fue el concepto de arquitectura. En palabras de Criado Boado (1999: 20), podemos decir que la misma es "[...] una tecnología de construcción del paisaje social que mediante dispositivos artificiales domestica el mundo físico", en tanto Tilley (1994: 17) señala que "es una creación deliberada del espacio, un hecho tangible y visible. Esto es porque las estructuras arquitectónicas juegan un rol fundamental en la creación y recreación, producción y reproducción de los espacios existentes y tienen profundos efectos estructurales en el espacio perceptivo [...]".

\section{USHNUS Y PLAZAS EN LA COSMOVISION INKAICA}

Las referencias etnohistóricas sobre el ushnu han sido estudiadas por varios investigadores, quienes han planteado diversas interpretaciones sobre su naturaleza (Zuidema 1980; Matos 1986; Hyslop 1990; Meddens 1997; Raffino 2004). Sin embargo, cabe resaltar las referencias que dan ciertos documentos etnohistóricos que: a) indican que estaban construidos en plazas y dentro de la red vial inkaica, así como las ofrendas de chicha que daban al sol en estos lugares (Albornoz 1967 [1582]: 24); b) enfatizan sobre determinadas ceremonias, dirigidas al sol, a las huacas, refiriéndose específicamente al Capac Hucha (Guamán Poma 1980 [1615]: 262 [264]) y, sobre todo, señalan que se trata de lugares de sacrificio y libación (Hernández Príncipe 1923: 63). Una de las primeras investigaciones sobre el ushnu fue realizada por Zuidema (1980), quien presenta una amplia y minuciosa información sobre el tema, desarrollando en sus investigaciones diversos planteamientos sobre la ideología inka, los cuales han servido a los posteriores investigadores como un marco referencial para entender el ushnu, sugiriendo que este tiene un carácter de acceso al "mundo subterráneo" (Zuidema 1980: 408), asociando la acción de "chupar" o absorber las ofrendas líquidas en los rituales realizados. Pero, a la vez, menciona que el ushnu en Cusco fue un centro de observaciones astronómicas y que posiblemente influyó en el trazo del plano general de esa ciudad (Zuidema 1980: 453).

Respecto de la ubicación del ushnu, no son claras las identificaciones del mismo en los sectores cercanos al Cusco, pero sí se han encontrado en territorios conquistados o en territorios que nada tienen que ver con la ocupación inkaica. La razón de esto no es clara, pero si tenemos en cuenta los comentarios de Betanzos, quien describe las principales diferencias con la plaza de Cusco y el Templo del Sol o qorikancha, vemos que solo la nobleza inkaica tenía acceso a este último, mientras que la plaza, en general, era usada para rituales a los cuales asistían las poblaciones comunes o no relacionadas con el Inka (Hyslop 1990).

Desde el plano estrictamente arquitectónico, estas estructuras se identifican como plataformas sobreelevadas, con escalinatas y accesos para ingresar a la parte superior de las plataformas, como también asientos o tianas en la cima de estas. Son generalmente de planta ortogonal con planos superpuestos (a modo de pirámides truncas) construidos en pirca. Además, presentan un lugar donde echar las ofrendas líquidas y su respectivo sistema de drenaje, siendo generalmente pozos de ofrenda o cocha, los cuales en su mayoría tenían al interior un relleno de 
piedras por donde se filtraba la ofrenda líquida (Matos 1986, 1994; Raffino et al. 1997; Pino Matos 2004). A su vez, el ushnu en la época inka no solo sería el lugar destinado a ofrendas líquidas, sino también se relacionaría con sacrificios de niños, animales y quema de tejidos y otras ofrendas (Hyslop 1990: 100), haciéndose más complejas las ceremonias y las formas de ofrendar.

A través de datos etnohistóricos se ha identificado que la aukaipata o plaza principal habría sido utilizada como un centro de aglomeración de grandes cantidades de personas. Las mismas eran amplias, de forma trapezoidal y en ellas se realizaban actividades religiosas y sociales. A su vez, el rito de la reciprocidad se efectuaba en la gran plaza, en donde los ayllu y linajes reales se reunían a comer, beber y bailar las danzas ceremoniales del calendario cusqueño (Garcilaso de la Vega 1963 en Hyslop 1990).

Dentro del registro arqueológico para el Noroeste Argentino podemos mencionar el sitio El Shincal, ubicado en el centro de la Provincia de Catamarca, a $60 \mathrm{~km}$ de Hualfín Inka. El mismo presenta una gran plaza central de importantes dimensiones con 175 metros de cada lado. En la periferia de la plaza se encuentran cuatro edificios rectangulares similares a aquellos que se clasifican como kallankas. Las nuevas interpretaciones que están surgiendo para dicho sitio lo vinculan con un nodo central que congregaba cantidades importantes de personas en la realización de fiestas y rituales estatales (Giovannetti 2009). Se han localizado en el cono aluvial del Quimivil una gran cantidad de conjuntos de molienda múltiple con muchos morteros en su superficie, siendo posible relacionarlo con el Período Inka de la región. Ha sido posible reconocer espacios de fogones cercanos al mortero donde los restos de cerámica y restos arqueobotánicos demostraron la producción de chicha a gran escala (Giovannetti 2009, Giovannetti et al. 2010).

Podemos decir que este espacio se constituía sobre todo para posibilitar y dar contexto a prácticas constitutivas y legitimadoras del poder del Estado y por ende de las elites (muchas veces elites locales que se plegaban al nuevo sistema de poder para mantener ciertos beneficios). Nielsen (2007) distingue también estos espacios no solo por la congregación frecuente de grandes grupos de personas, sino además en el plano andino, las acciones desarrolladas en las mismas podrían mostrar una estrecha y explícita relación con la imagen de la comunidad sobre sí misma, las autoridades y el conjunto de valores apropiados y defendidos. Las plazas de los sitios Los Amarillos y Lakaya (Lípez) les han demostrado la centralidad del culto a los antepasados en grupos de jerarquías corporativas y descentradas políticamente. Las plazas cumplirían un rol fundamental en la unión de la comunidad integrándola en el mismo espacio compartido (Nielsen 2006). Pero, como muy bien lo señala el autor, la hawkaipata inka pone en primer plano la institución del Estado más que la representación de los ancestros, aunque el poder político pueda recurrir aún a discursos sobre el pasado, pero ya con significados de carácter panandino más que localizadas prácticas de comunidades concretas. Moore (1996), por su parte, ha discutido comparativamente el significado, desde el punto de vista de las relaciones sociales jerárquicas, de este tipo de manifestaciones espaciales. Es decir que, en general, las plazas en los Andes fueron el espacio para los rituales principales combinando discursos, música, danza y despliegues en ceremonias que fusionaban comunidades, legitimaban distinciones y jerarquía social y consolidaban las creencias cosmogónicas. Moore (1996) destaca de las plazas inkaicas que involucraban grandes grupos de gente en rituales comunitarios a diferencia de otro tipo de sociedades andinas como los Chimú o los Tiwanaku.

Por último, cabe resaltar que en los territorios conquistados pueden encontrarse dichas plataformas, unificando la idea anteriormente mencionada de la nobleza inkaica (en donde el Inka se sentaba sobre ellas) con las grandes plazas, asociado a la gente común. Esto, a su vez, estaría estrechamente vinculado con el hecho de la legitimación del poder del Inka sobre las poblaciones conquistadas.

\section{BREVE DESCRIPCIÓN DEL SITIO HUALFIN INKA}

El sitio Hualfín Inka se encuentra emplazado sobre una terraza aluvial en el centro-oeste de la Provincia de Catamarca, Argentina, cuyas coordenadas son $27^{\circ} 13^{\prime} 46^{\prime \prime}$ de latitud sur y 66\%48'55" de longitud oeste (fig. 3). Se encuentra flanqueado por sierras de hasta $3500 \mathrm{~m}$, pertenecientes a las geológicamente denominadas Sierras Pampeanas Noroccidentales (González Bonorino 1950: 89).

En la región predomina un clima árido o semiárido, donde las aguas superficiales son muy escasas. Los ríos y arroyos son generalmente de poco caudal y de régimen transitorio (Caminos 1979: 282). El río Hualfín es, generalmente, un curso de poco caudal en los meses de temporada seca (abril-noviembre) y aumenta en temporada húmeda, los meses de verano (diciembremarzo). La vegetación está compuesta principalmente por jarillas, cardones, chañares y, cercanos a la población local, algarrobos (Lynch et al. 2006: 198).

El primer registro que se tiene del sitio es entre los años 1896 y 1897, cuando el entomólogo C. Bruch 


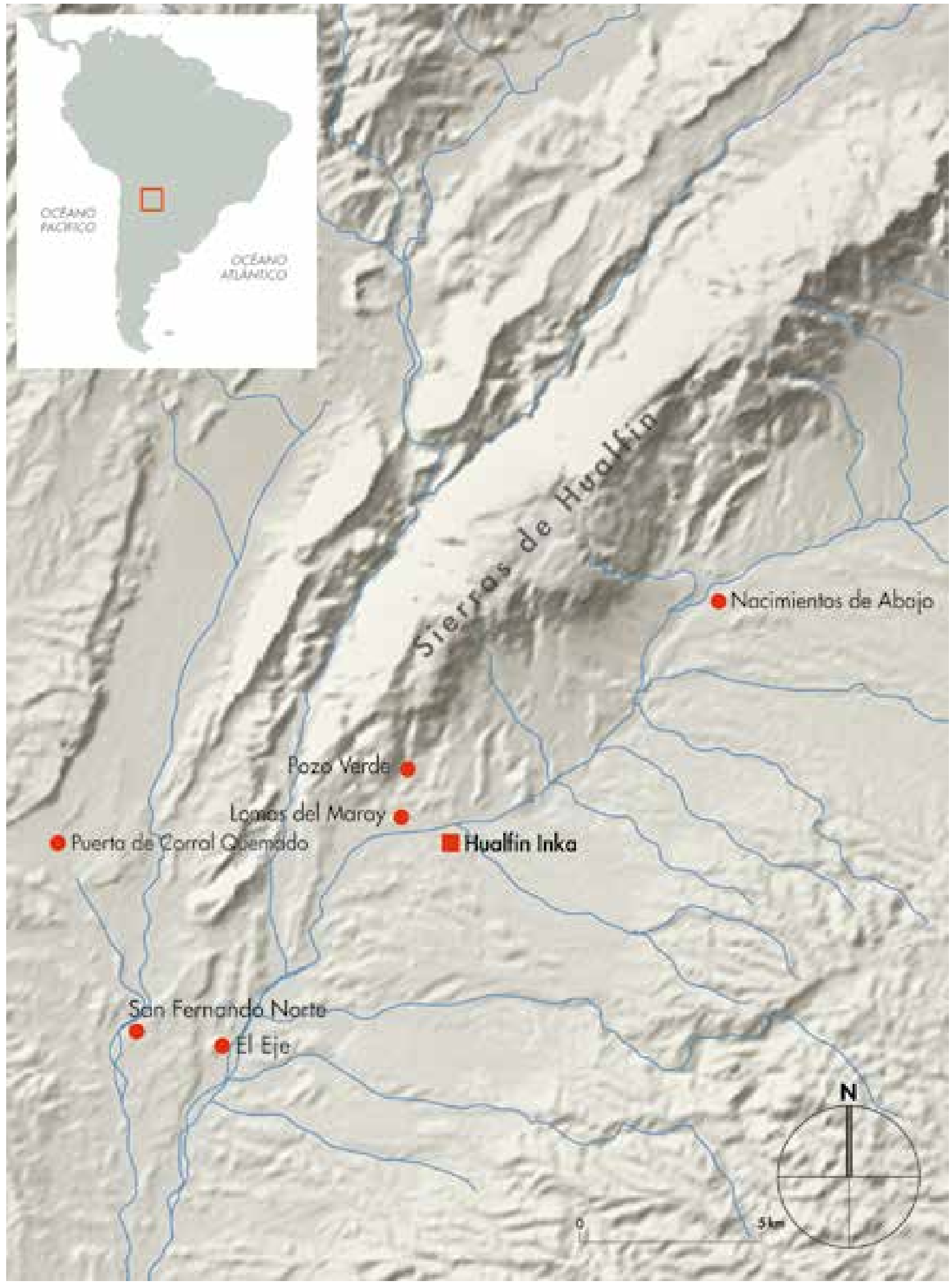

Figura 3. Ubicación del sitio arqueológico Hualfín Inka, Valle de Hualfín, Catamarca.

Figure 3. Location of Hualfin Inka archeological site in the Hualfin Valley, Catamarca. 
es contratado por F. P. Moreno como naturalista del Museo de La Plata, Argentina, y enviado a la localidad de Hualfín con la misión de realizar colecciones de la zona. A su regreso publica el primer croquis del sitio a mano alzada y sin escala (Bruch 1904). En la década de 1980, Raffino y colaboradores (1982) realizaron un nuevo plano del emplazamiento y una representación arquitectónica tentativa del mismo, sin intervención arqueológica, pudiendo comprobar que la organización de los espacios respondía al patrón urbano inkaico.

Investigaciones recientes llevadas a cabo por la autora de este trabajo dieron cuenta de características arquitectónicas particulares en las que se combina una organización del espacio de acuerdo con los patrones estatales con una técnica constructiva de carácter local. En este sentido, los muros fueron levantados con rocas subangulares a subcuadrangulares dispuestas en doble hilera, con relleno de ripio, y si bien no se ha hallado roca canteada, se ha podido constatar una selección de las caras más planas dispuestas hacia el exterior de los recintos. (Lynch et al. 2007, Lynch 2010).

Cabe agregar que para el valle de Hualfín, el período de Desarrollos Regionales (900/1000-1470 DC) se manifestó en grupos asentados en todo el valle en diversos ambientes, portadores de la denominada Cultura Belén, conocida principalmente por la cerámica Belén Negro sobre Rojo. Gran parte de los sitios de habitación se hallaban sobre lomas de difícil acceso, muchas de ellas protegidas por murallas defensivas, de diferentes dimensiones y con distintos grados de aglomeración (Balesta \& Wynveldt 2010). Por su parte, los asentamientos inkaicos identificados para el Noroeste Argentino se distribuyen sobre terrazas fluviales disponibles en el fondo del valle. Es por ello que los sitios del Tawantinsuyu se constituirían en verdaderos enclaves espaciales, núcleos desde los que se establecería la inkaización de las poblaciones locales (Sánchez 2004) y que se articularían por medio del trazado de la red vial.

Para sistematizar el estudio del sitio, este fue dividido en tres sectores, A, B y C, cuyas altitudes difieren entre 15 y 20 m entre la zona más baja y la más alta (Raffino et al. 1982: 481), los cuales fueron retomados en la planimetría y la descripción del sitio realizado por Lynch (2010) (fig. 4). El sector A se ubica hacia el noreste, tiene una superficie de aproximadamente $15.000 \mathrm{~m}^{2}$ con forma de polígono irregular y está delimitada por un muro perimetral. Esta estructura es una plaza intramuros o aukaipata inkaica, con estructuras adosadas en su interior (kallanka, torreón, ushnu) (fig. 5).

El sector B contiene un conjunto de tres kanchas, situados aproximadamente en línea, que poseen recintos rectangulares y circulares. La planta de estos conjuntos es irregular, circunstancia que no parece responder a un condicionamiento topográfico, puesto que la superficie no es especialmente abrupta. En el grupo de construcciones intermedias se encuentran cuatro recintos rectangulares, uno de los cuales, de dimensiones mayores, correspondería a otra kallanka.

El sector C se localiza al suroeste de la plaza y se compone de un grupo de cuatro Rectángulos Perimetrales Compuestos (RPC), de perímetro más regular que los del grupo B y tres grupos de recintos circulares agrupados, que fueron interpretados como collcas (Raffino et al. 1982: 481; Lynch 2010).

\section{AUKAIPATA Y USHNU DE HUALFÍN INKA}

Durante los trabajos de campo realizados desde el 2005 hasta la actualidad se ha podido diferenciar una sola ocupación perteneciente al momento inkaico. Esto se encuentra corroborado no solo por los trabajos de excavación, que se expondrán más adelante, sino por fechados absolutos realizados con la técnica de AMS sobre carbón vegetal, en el cual los fechados calibrados evidencian un momento inkaico entre los años 1300 y 1477 DC (límites máximos). ${ }^{2}$

Respecto del ushnu del sitio, el mismo se encuentra situado en el sector sureste de la plaza intramuros. Se trata de una plataforma artificial de, por lo menos, un metro de altura, de forma rectangular, de 3,5 por $7,5 \mathrm{~m}$. Está separada del muro perimetral unos $4 \mathrm{~m}$ y presenta un acceso por una escalera de, al menos, tres peldaños sobre la pared, con una orientación suroeste que mira hacia la plaza (Lynch et al. 2007, Lynch 2010). Los muros son dobles, con un espesor que oscila entre los 0,50 y $0,60 \mathrm{~cm}$, tienen un cementante compuesto de barro y ripio y no presentan caras canteadas, aunque sí podría haber una selección de las caras más planas dispuestas hacia el exterior (fig. 6).

La excavación produjo el hallazgo de una variedad de materiales arqueológicos entre los que se cuentan aquellos cerámicos, óseos, líticos y arqueobotánicos. Asimismo, si bien cuantitativamente escasos, también se identificaron restos de naturaleza malacológica, como es el caso de pequeñas cuentas de nácar. Un dato remarcable es la relativa abundancia de material recuperado en comparación al resto de las estructuras excavadas. En estas últimas el registro fue escaso respecto de la superficie total de excavación (Lynch et al. 2010). Se detallan a continuación las características sobresalientes de las distintas ergologías recuperadas. 


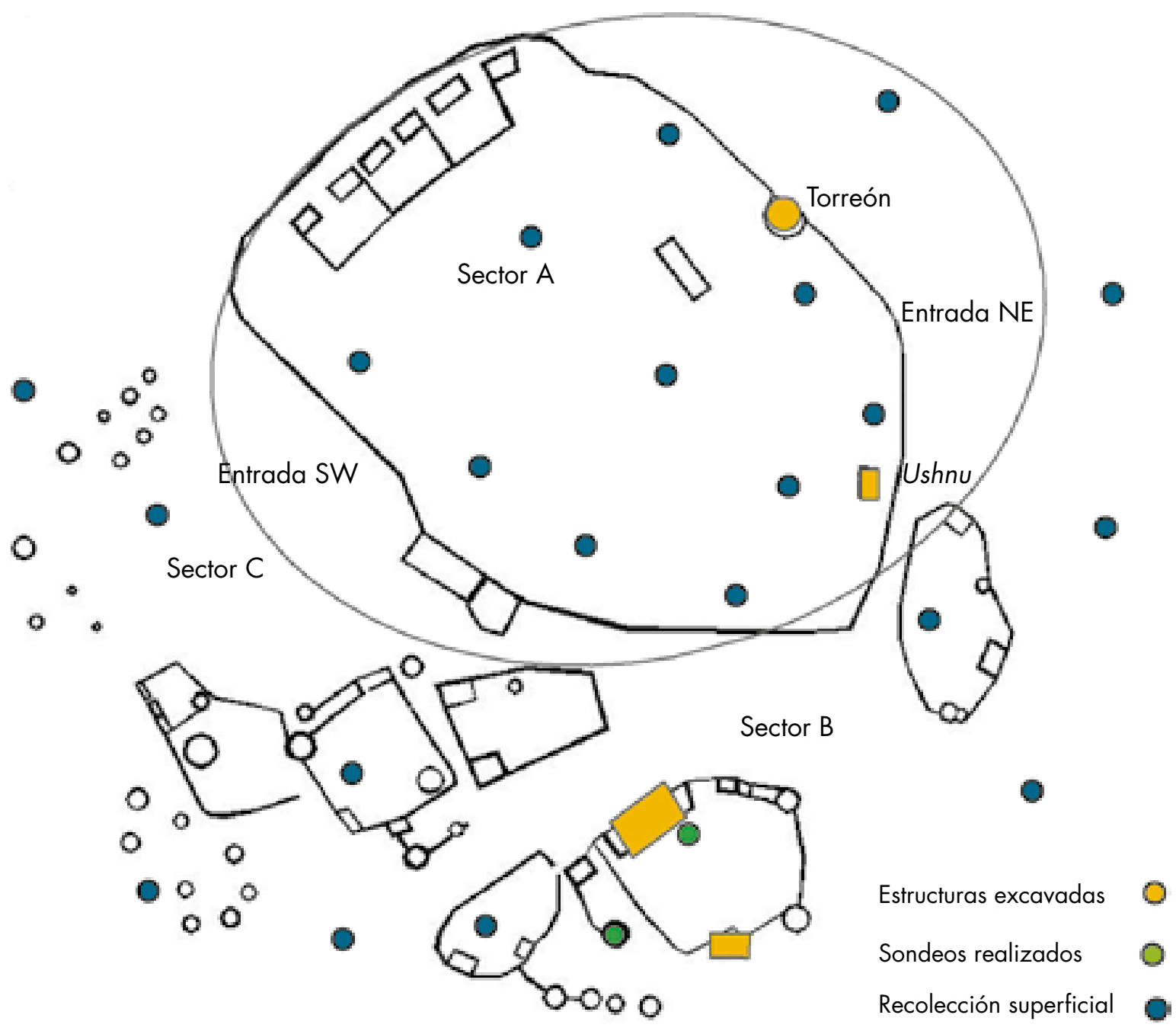

Figura 4. Plano del sitio Hualfín Inka registrado con estación total en donde se registran las actividades realizadas en los distintos sectores del sitio. Se señala las entradas a la plaza que estarían relacionadas con el camino inkaico proveniente del NE.

Figure 4. Site plan of Hualfin Inka recorded via total station survey, indicating the activities carried out in different sectors of the site. The layout shows the entrance to the plaza where the Inka Road arrived from the NE.

En cuanto al material cerámico, el conjunto total de materiales recuperados en una superficie de $8 \mathrm{~m}^{2}$ es de 71 fragmentos ( $\mathrm{n}=71$ ). Dentro de ellos, es proporcionalmente importante la presencia de estilos decorativos asociados al Período Tardío o de Desarrollos Regionales (900-1470 DC), en relación con los inkaicos. En este sentido, hemos podido constatar un mayor porcentaje de los estilos locales denominados Belén y Santa María, mientras que el Estilo Inka (1470-1532 DC) se encuentra presente tan solo en un $7 \%$ del total de la muestra obtenida. Si bien la cantidad de material cerámico asociado al momento inkaico es inferior en comparación a los estilos locales, esto pondría en evidencia la hipótesis planteada de una ocupación poco intensa, en la que las prácticas estatales habrían tenido una escasa relevancia (Lynch 2010). Esto contrasta notablemente con una arquitectura planificada de acuerdo con los cánones propios del Tawantinsuyu. Es muy probable que el sitio haya perdido importancia con el correr del tiempo, momento en el cual alcanza su máxima expresión el sitio El Shincal.

Los materiales de tipo tosco sin decoración son, sin embargo, los más numerosos (Lynch \& Páez 2009). Asociado a este material cerámico se encontraron restos óseos de animales, familias de Camelidae y Cervidae (41\%), (Lynch et. al 2007, Lynch 2010); artefactos líticos correspondientes a lascas, lascones, núcleos y mayormente desechos de talla. Las distintas materias primas que componen el conjunto incluyen basalto, 


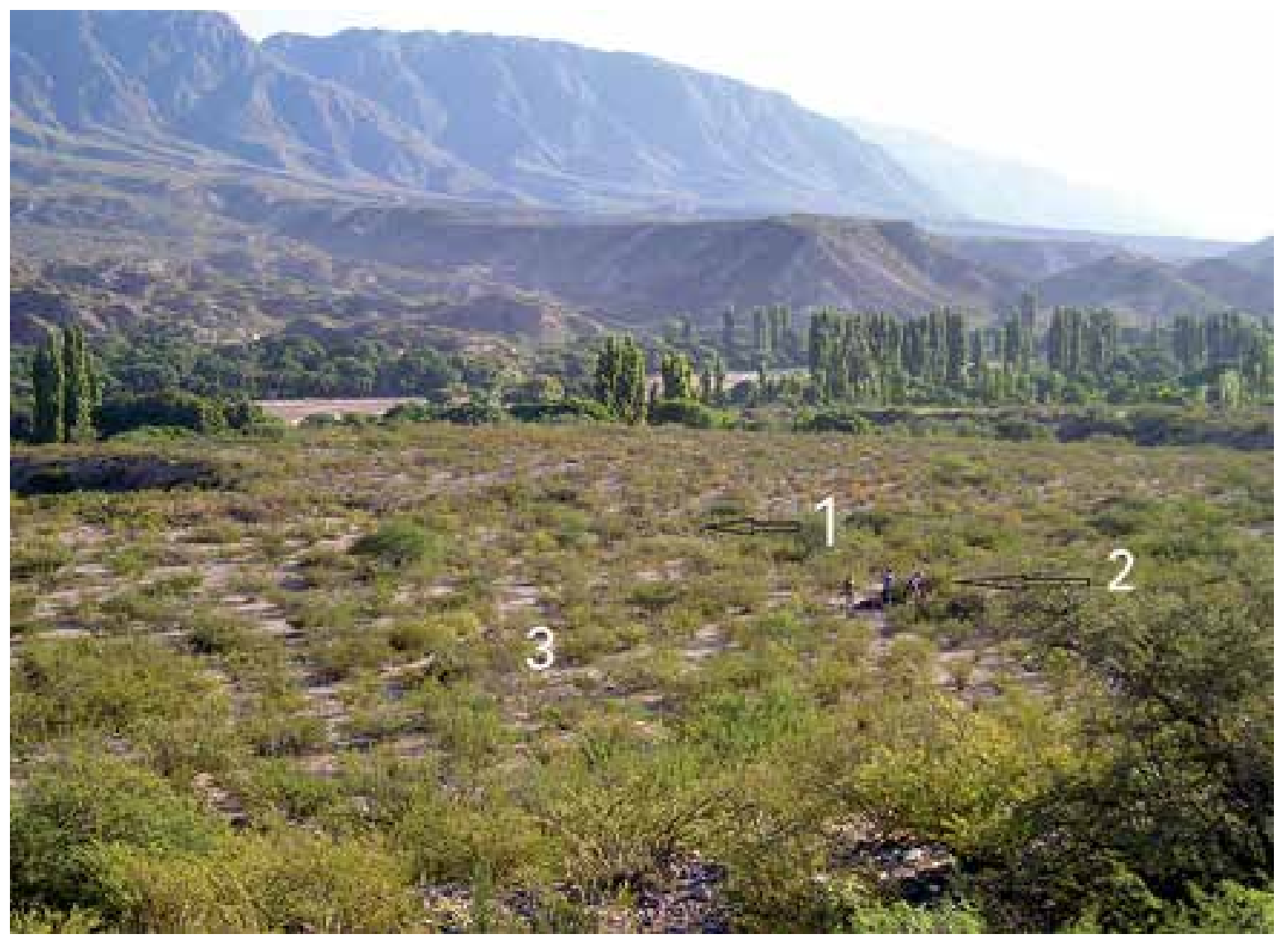

Figura 5. Vista panorámica de la plaza desde el sector B indicando: 1) muro perimetral; 2) el ushnu; 3) la plaza misma. Figure 5. Panoramic view of the plaza from sector B, indicating: 1) the perimeter wall; 2) the ushnu; 3) the plaza itself.

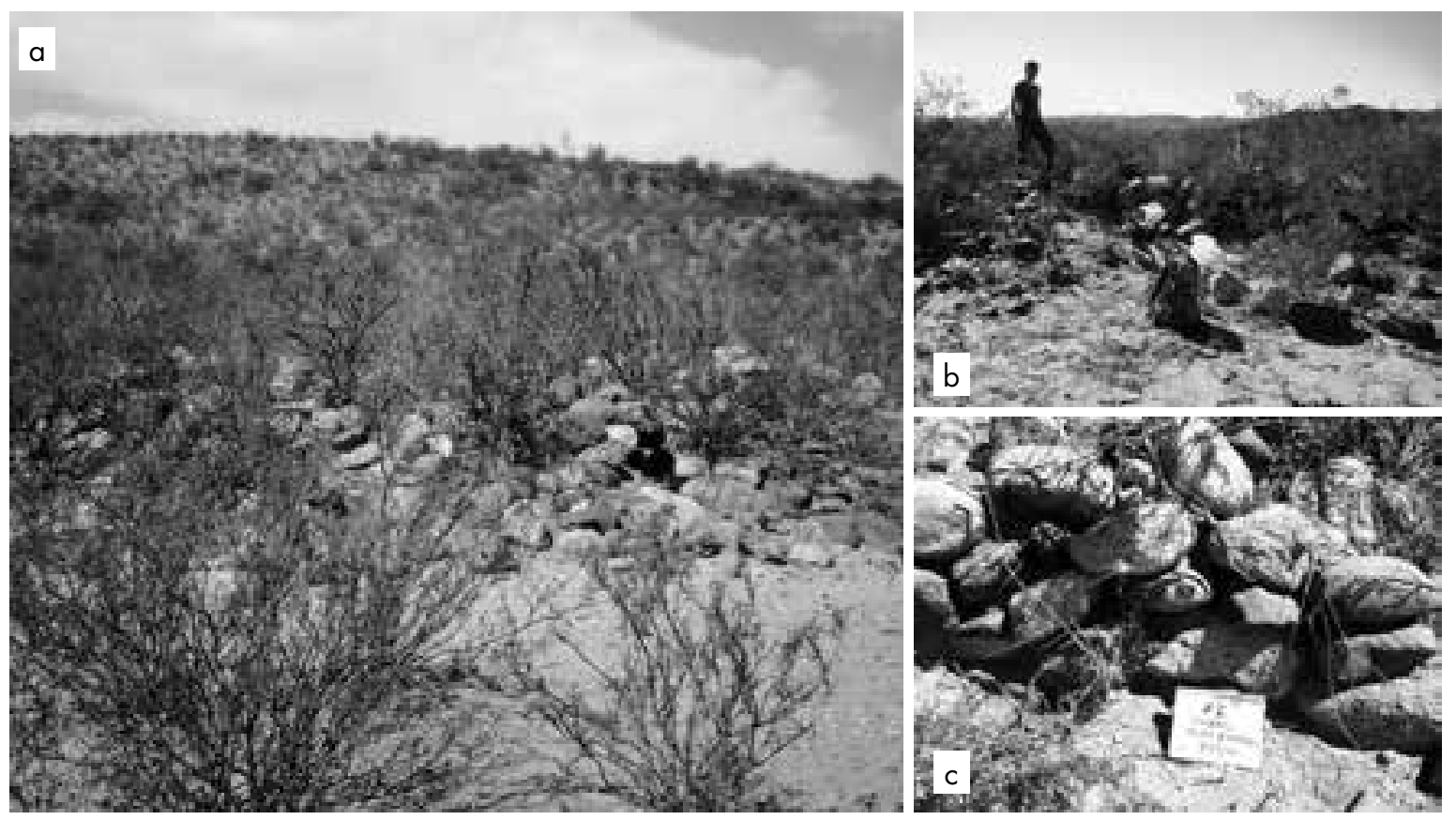

Figura 6. a y b) Dos perspectivas del ushnu de Hualfín Inka visto desde la plaza; c) Detalle de los muros.

Figure 6. $a \& b$ ) Two views of the ushnu of Hualfin Inka, seen from the plaza; c) Detail of the wall. 
cuarzo, cuarcita, sílices de distinta coloración y arenisca (Lynch 2010: 36, Lynch \& Lynch 2010: 1687) (figs. 7, 8 y 9). El registro arqueobotánico recuperado cuenta con una importante abundancia de carbones de diferentes tamaños, diferenciándose entre ellos restos de maíz (Zea mays) (Lynch 2010). Dentro de las plantas cultivadas, destacamos el hallazgo de un grano de poroto (Phaseolus vulgaris). Con una incidencia muy baja se identificaron también restos de vegetales silvestres como chañar (Geoffroea decorticans) y algarrobo (Prosopis sp.). De cualquier forma, los restos arqueobotánicos más numerosos fueron fragmentos de leños carbonizados, lo que evidencia una destacable presencia de fogones dentro de esta estructura arquitectónica. De aquí se obtuvo un fechado radiocarbónico (AA 85878/Cuad. S2, fogón, Niv.12, 0,90 cm, carbón vegetal), dando una edad radiocarbónica de 515 \pm 42 AP.

Respecto de la plaza ubicada hacia el noreste del sitio, la misma está rodeada de un muro perimetral, ocupando una superficie de $15.000 \mathrm{~m}^{2}$. En ella se realizó una

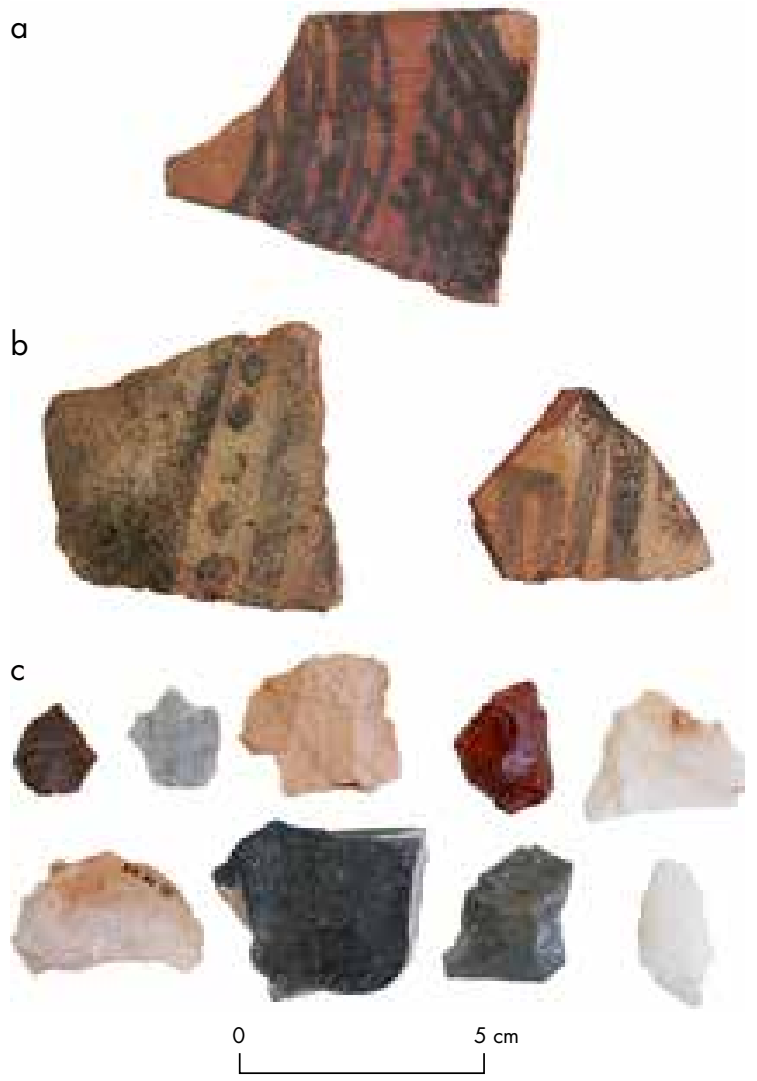

Figura 7. a) Material cerámico Belén Negro sobre Rojo; b) Material cerámico Santamariano; c) Material lítico del ushnu.

Figure 7. a) Ceramic material from the Black on Red Belén tradition; b) Ceramic material from the Santamariano tradition; c) Lithic material from the ushnu. recolección superficial tanto de material cerámico como lítico, dando como resultado numerosos fragmentos de cerámica diagnóstica $(n=650)$ atribuibles al estilo local Belén Negro sobre Rojo (24\%) y en menor proporción fragmentos Santamarianos (4\%), ambos estilos característicos del Período Tardío o de Desarrollos Regionales (900/1000-1470 DC) como así también fragmentos atribuibles al Estilo Inka Provincial (20\%) (1470-1532 DC) (fig. 10).

En cuanto al material lítico superficial, este se encuentra representado por materias primas de la zona. Puede decirse que la materia prima más abundante es el basalto, pero también se encuentran el cuarzo, la cuarcita, la sílice de diferentes variedades, la calcedonia y el xilópalo. Con relación al análisis tecnomorfológico, los materiales fueron diferenciados en dos grandes grupos, por un lado, los artefactos formatizados (AF) y, por el otro, los no formatizados (ANF). A su vez fueron consideradas diferentes variables para describir sus características morfométricas (Aschero 1975, 1983). Los materiales recuperados superficialmente corresponden a un total de 49 artefactos líticos no formatizados (de ahora en más ANF), 29 desechos de talla (DT) y seis núcleos (NUC), dentro de los cuales cuatro corresponden a una roca basáltica de color grisáceo; mientras que los dos restantes pudimos identificarlos dentro de la categoría de sílex del mismo color que los anteriores (figs. 11 y 12).

En el sector A se encuentran otras estructuras adosadas al interior de la plaza que detallaremos a continuación. En el sector noroeste de la plaza se encuentra incluido un RPC o kancha, construcción característica inkaica, que ocupa una superficie aproximada de $1840 \mathrm{~m}^{2}$. Se ingresa al interior desde la plaza a través de una abertura, que hemos definido como puerta de acceso, de $6 \mathrm{~m}$ de ancho. La misma comunica a un pasillo de unos $70 \mathrm{~m}$ de largo encerrado entre muros. Al final del pasillo se puede ver una entrada entre dos recintos, a través de la cual se ingresa a una serie de cuatro sectores colocados en línea y comunicados entre sí por accesos de unos $2 \mathrm{~m}$ de ancho.

Estos recintos fueron identificados funcionalmente como patios (Raffino 1982), sin embargo, no hemos realizado excavaciones en los mismos, ya que presentan un gran deterioro de las estructuras debido principalmente a factores antrópicos. Igualmente hemos podido determinar sus medidas, ya que todavía se encuentran los cimientos de las estructuras; estas son $17 \mathrm{~m}$ de ancho por un largo que varía entre los $16 \mathrm{~m}$ y los $22,5 \mathrm{~m}$. Incluidos en los patios y adosados al muro que da al pasillo se verifican dos recintos cuyas entradas dan al patio, cuyas medidas son prácticamente idénticas: 8 por 


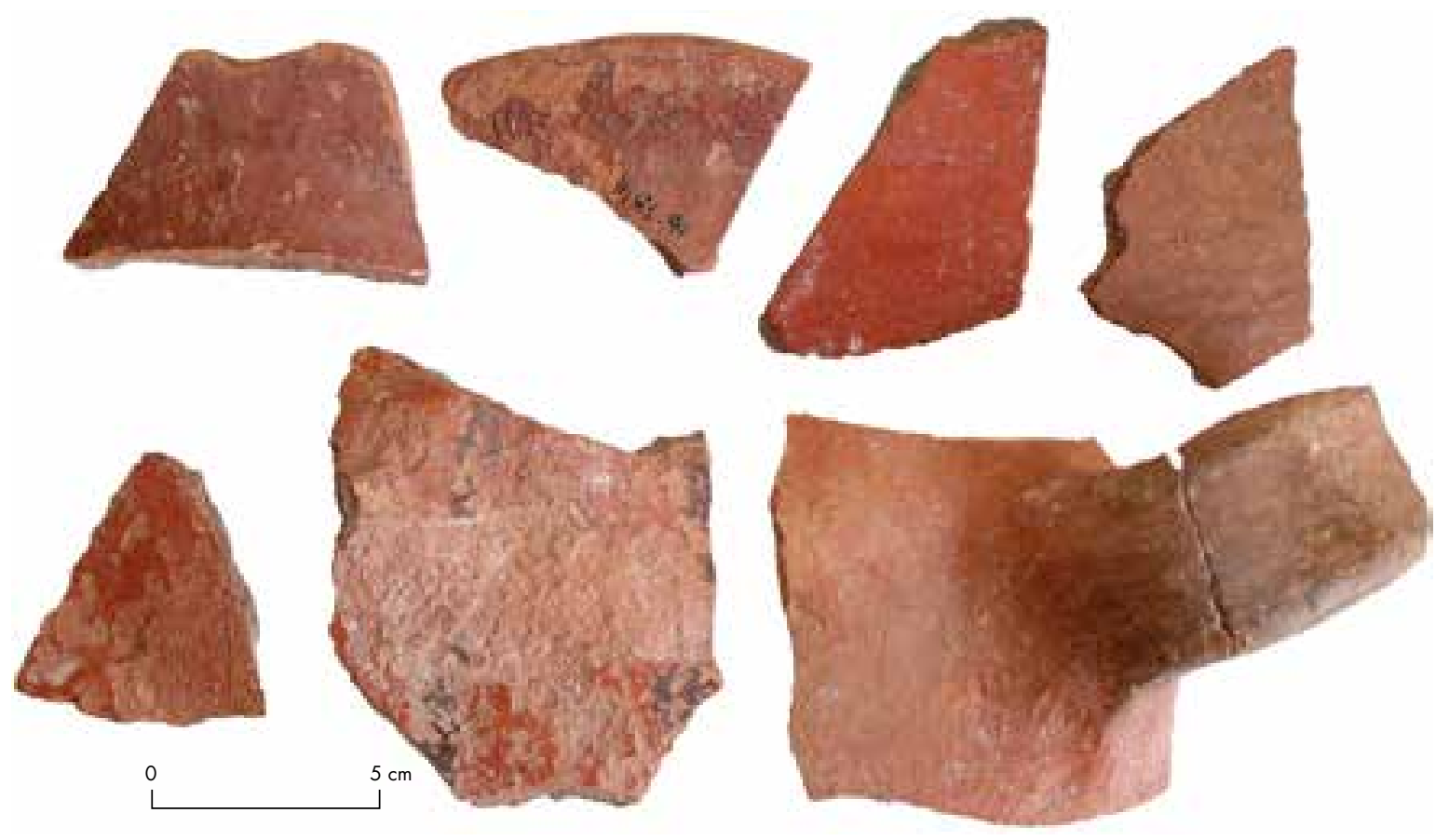

Figura 8. Material cerámico inkaico del ushnu.

Figure 8. Inka ceramic material from the ushnu.
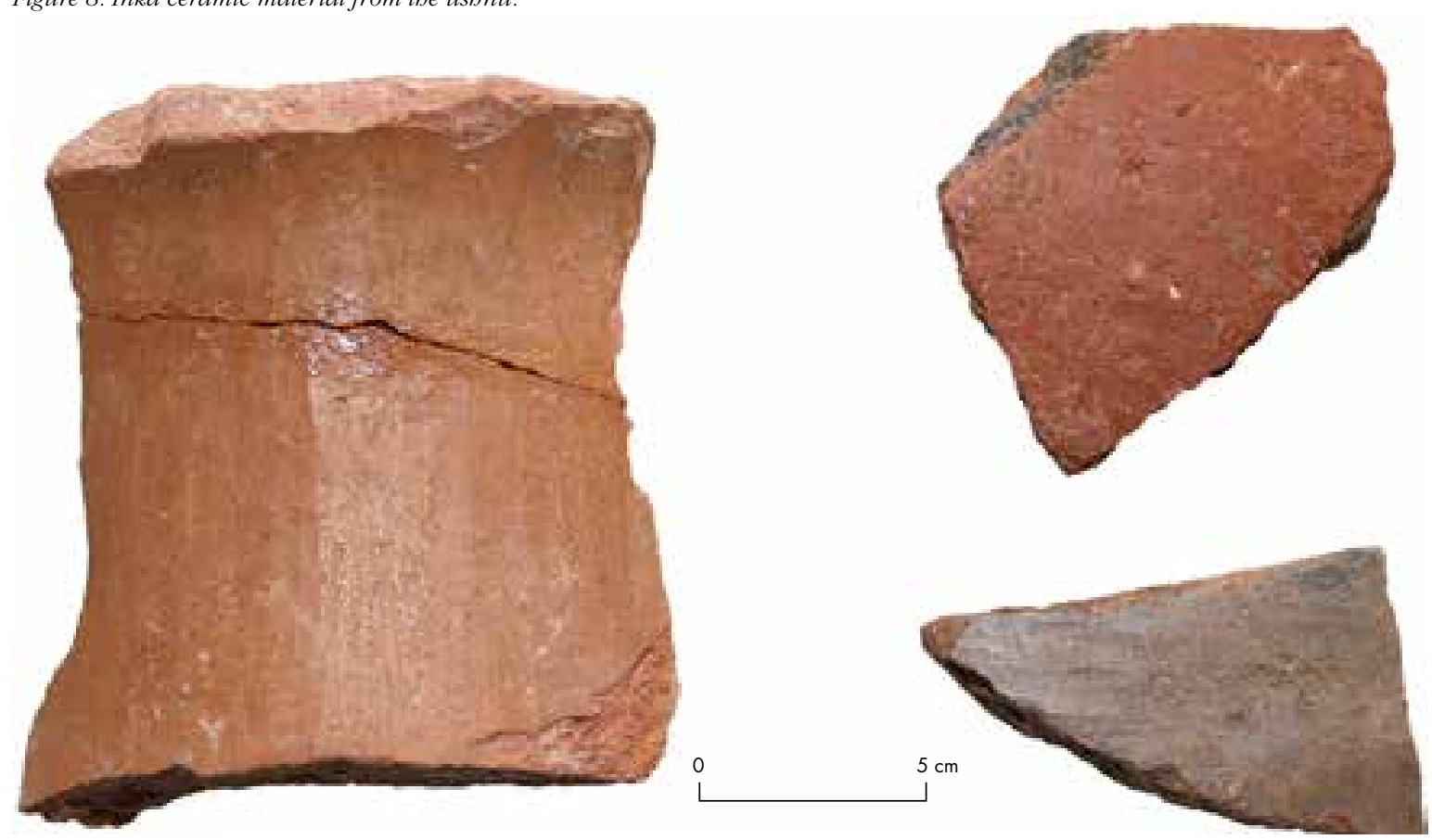

Figura 9. Material cerámico superficial inkaico de la plaza.

Figure 9. Inka ceramic material found on the surface inside the plaza. 
5,50 m. Todo el conjunto ofrece la idea de una tendencia hacia la edificación en damero regular, con simetría en la distribución del espacio y la forma, siendo la orientación de todo este sector noreste-suroeste (Lynch 2010).

Adosado al muro noreste de la plaza se observa un recinto de planta circular de 9,50 m de diámetro,

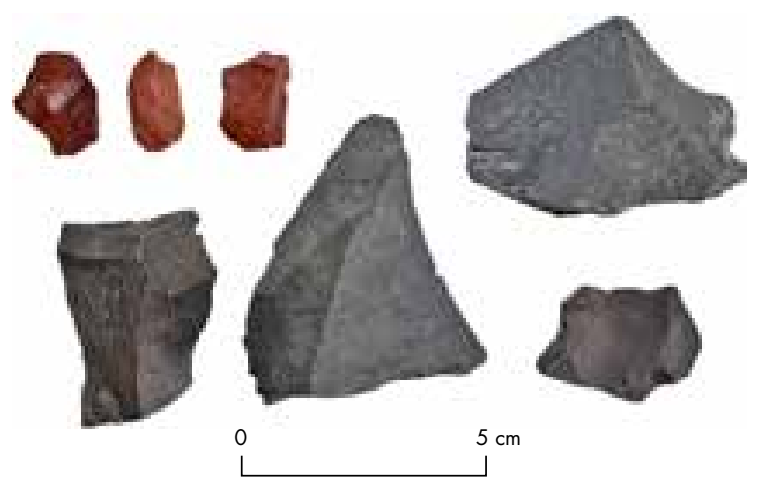

Figura 10. Material lítico superficial de la plaza: lascas de basalto y sílice.

Figure 10. Lithic material (basalt and silica flakes) found on the surface inside the Plaza. cuya entrada mira hacia la misma. Las características de ubicación y construcción de este recinto dan una imagen similar a los clásicos torreones inkas (Raffino et al. 1982: 118).

Finalmente, adosado a la pared suroeste de la plaza, se encuentra un recinto de planta rectangular, de 7 por $19 \mathrm{~m}$ de longitud. Por sus características arquitectónicas ha sido definido como una kallanka, que al igual que el ushnu es otra forma característica de la arquitectura inkaica. La fachada de este recinto es una prolongación del muro perimetral, mientras que la construcción en sí se encuentra por fuera del perímetro. El mismo se encuentra sobreelevado alrededor de un metro respecto del nivel de la plaza, presenta tres entradas en el frente, a las que se accede desde aquella, por otras escalinatas encerradas por un doble muro reforzado. Este doble muro estaba construido con bloques de piedra de color blanco-grisáceo y rosa-morado, posiblemente en hiladas alternadas. Como ocurre en el sitio El Shincal, a tan solo $60 \mathrm{~km}$ de distancia, el techo de esta kallanka habría sido a dos aguas o en mojinete. En su fachada es visible un intento de levantar muros en sillería, por

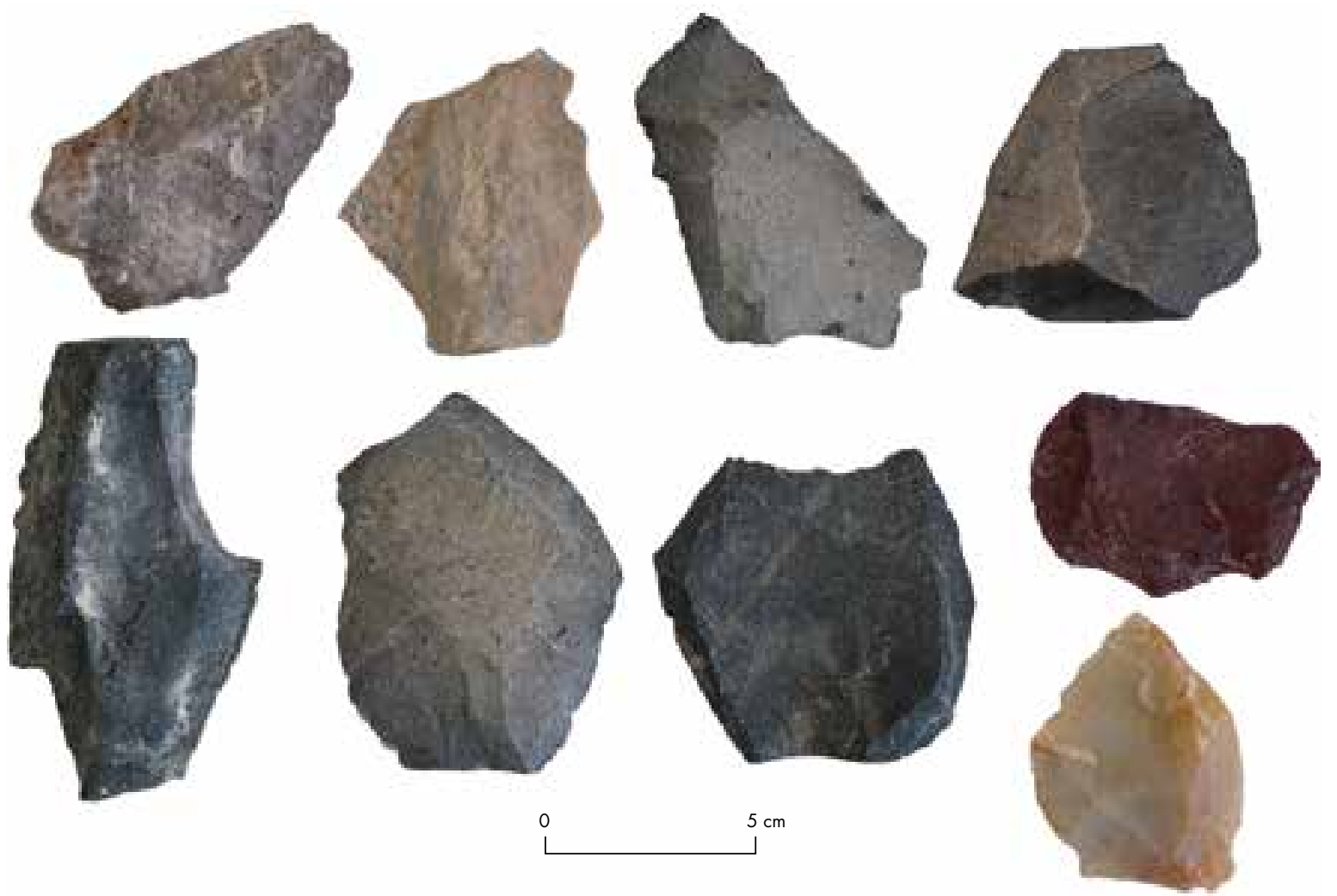

Figura 11. Material lítico superficial de la plaza: lascas de basalto.

Figure 11. Lithic material (basalt flakes) found on the surface inside the plaza. 


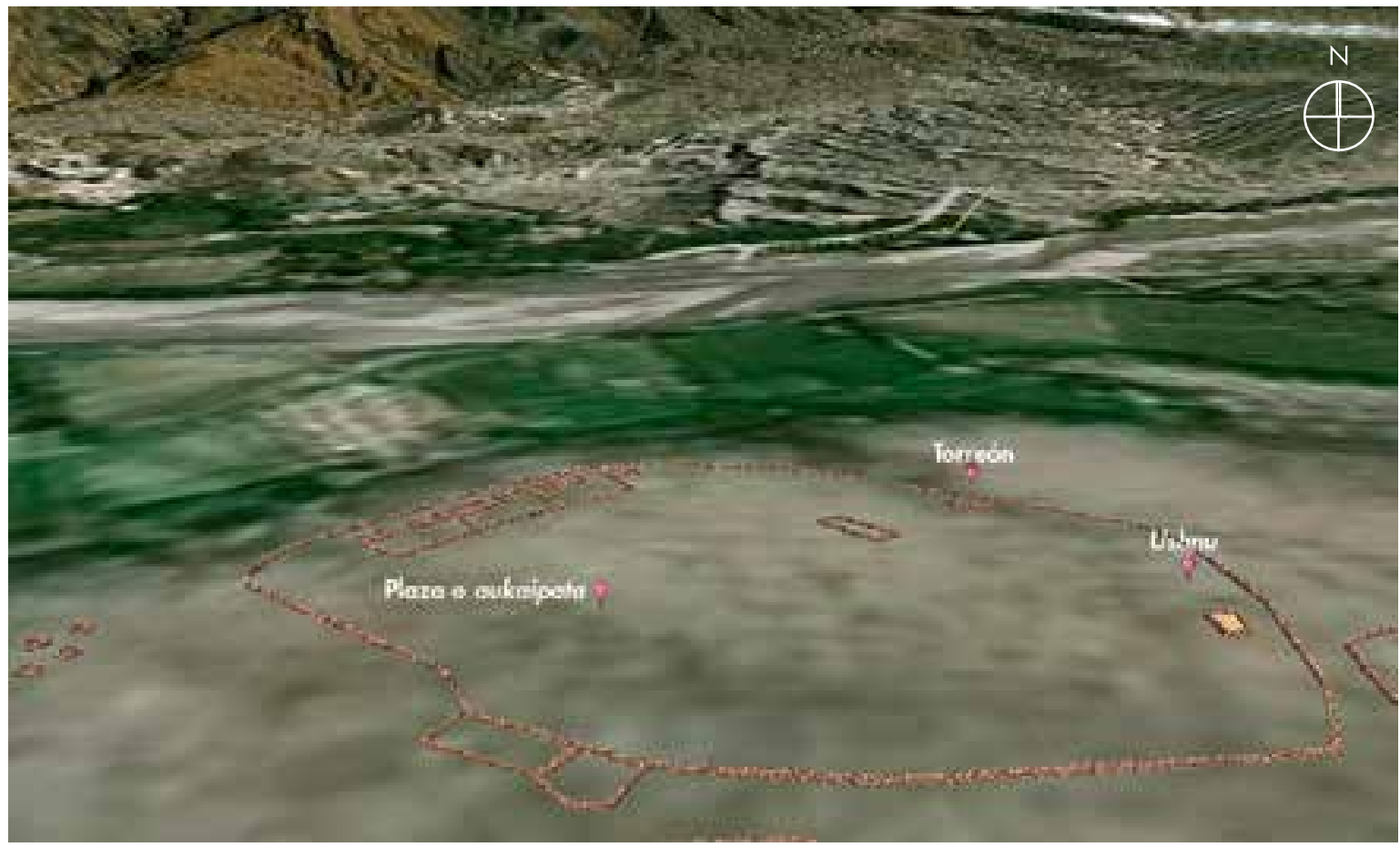

Figura 12. Perspectiva de la plaza junto con las estructuras internas asociadas. Figure 12. Outline of the plaza with its associated internal structures.

medio de una selección de la piedra y la disposición de su cara más regular y plana hacia el exterior. Esto estaría evidenciado en casi la totalidad de los recintos que componen el sitio (Lynch 2010).

Vemos que todo el sector A anteriormente descrito sería el de mayor relevancia del sitio, debido no solo a su ubicación en el espacio, sino a su arquitectura y los contextos arqueológicos encontrados, ya que en general son recintos que denotan una mayor dedicación en su planificación y construcción, que indudablemente tendrían importancia desde el punto de vista político, administrativo e incluso religioso. Este tipo de actividades, cuyo fin último sería la legitimación del poder por parte del Estado cusqueño sobre las poblaciones locales y perpetuar las jerarquías sociales por medio del ritual y de la fiesta, se ven a lo largo de los emplazamientos construidos en el Noroeste Argentino, viéndose una gran variabilidad de tipos de estructuras, como así también de la materia prima utilizada.

Cabe resaltar que, de acuerdo con los hallazgos y los fechados radiocabónicos efectuados en Hualfín Inka (Lynch 2010), las prospecciones realizadas alrededor del sitio y teniendo en cuenta sus características arquitectónicas, las estructuras parecen ser enteramente inkaicas, pudiendo argumentar que el sitio estuvo poblado durante la presencia del Estado relacionándose directamente con las poblaciones locales.

\section{DISCUSIONES Y CONCLUSIONES}

Hasta aquí hemos analizado la arquitectura, el tipo de emplazamiento y el registro material encontrado tanto en superficie como en estratigrafía de las dos estructuras más importantes del sitio, la aukaipata o plaza intramuros y el ushnu.

Vemos que todo el sector A respondería a una organización arquitectónica de tipo central, es decir, una composición estable y concentrada, compuesta de numerosos espacios secundarios que se agrupan en torno a uno central, dominante y de mayor tamaño (la plaza o aukaipata). El espacio central y unificador de la organización generalmente es de forma regular y de dimensiones lo suficientemente grandes que permitan reunir a su alrededor a los espacios secundarios. Como respuesta a sus respectivas exigencias funcionales, a su importancia con relación al conjunto, o al mismo contexto, los espacios secundarios pueden diferir formalmente entre sí, situación que posibilita la adecuación de la forma organizativa a las distintas características de su emplazamiento (figs. 13 y 14). 


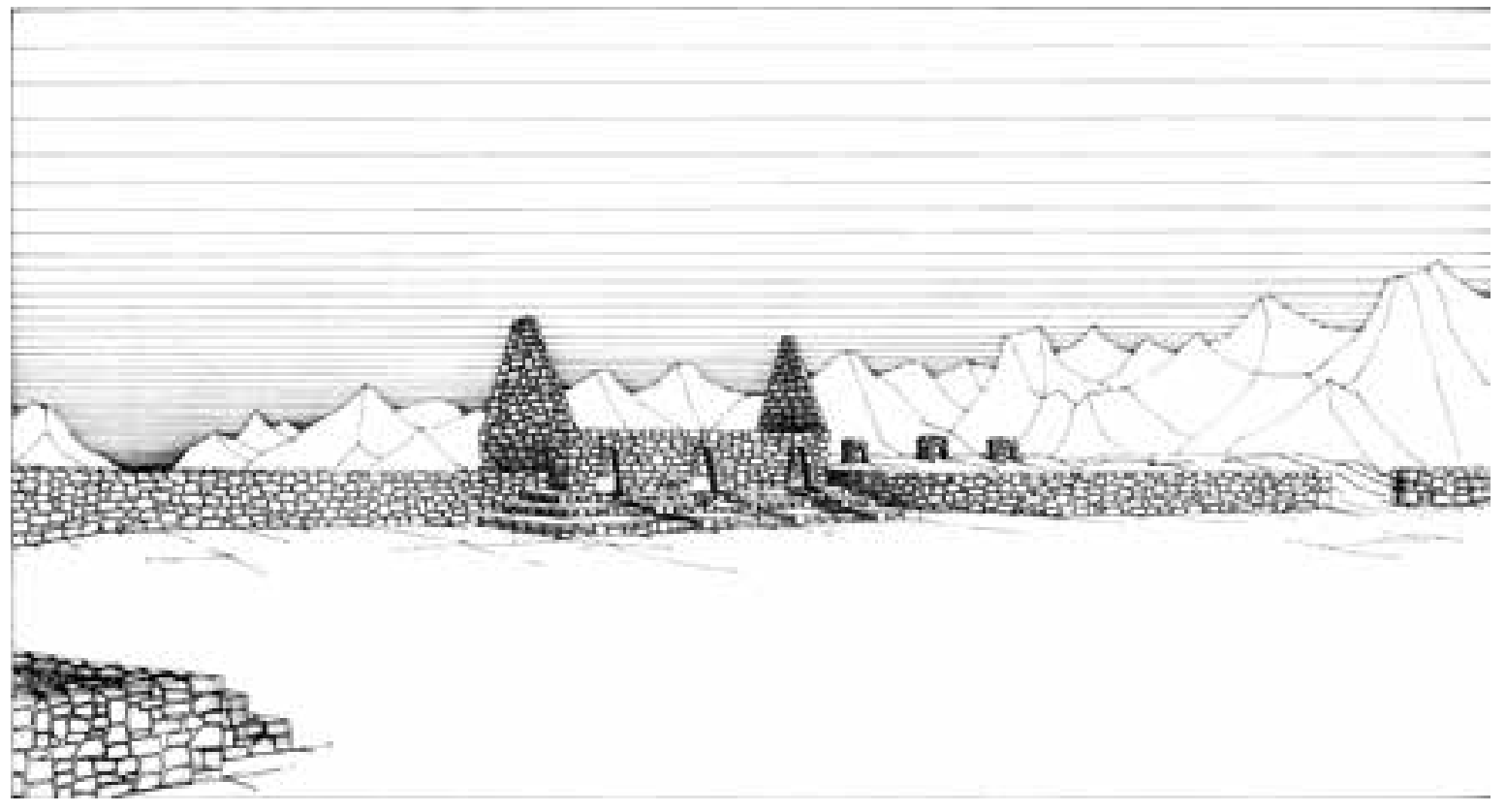

Figura 13. Perspectiva desde un sector de la plaza.

Figure 13. Recreated view from one sector of the plaza.

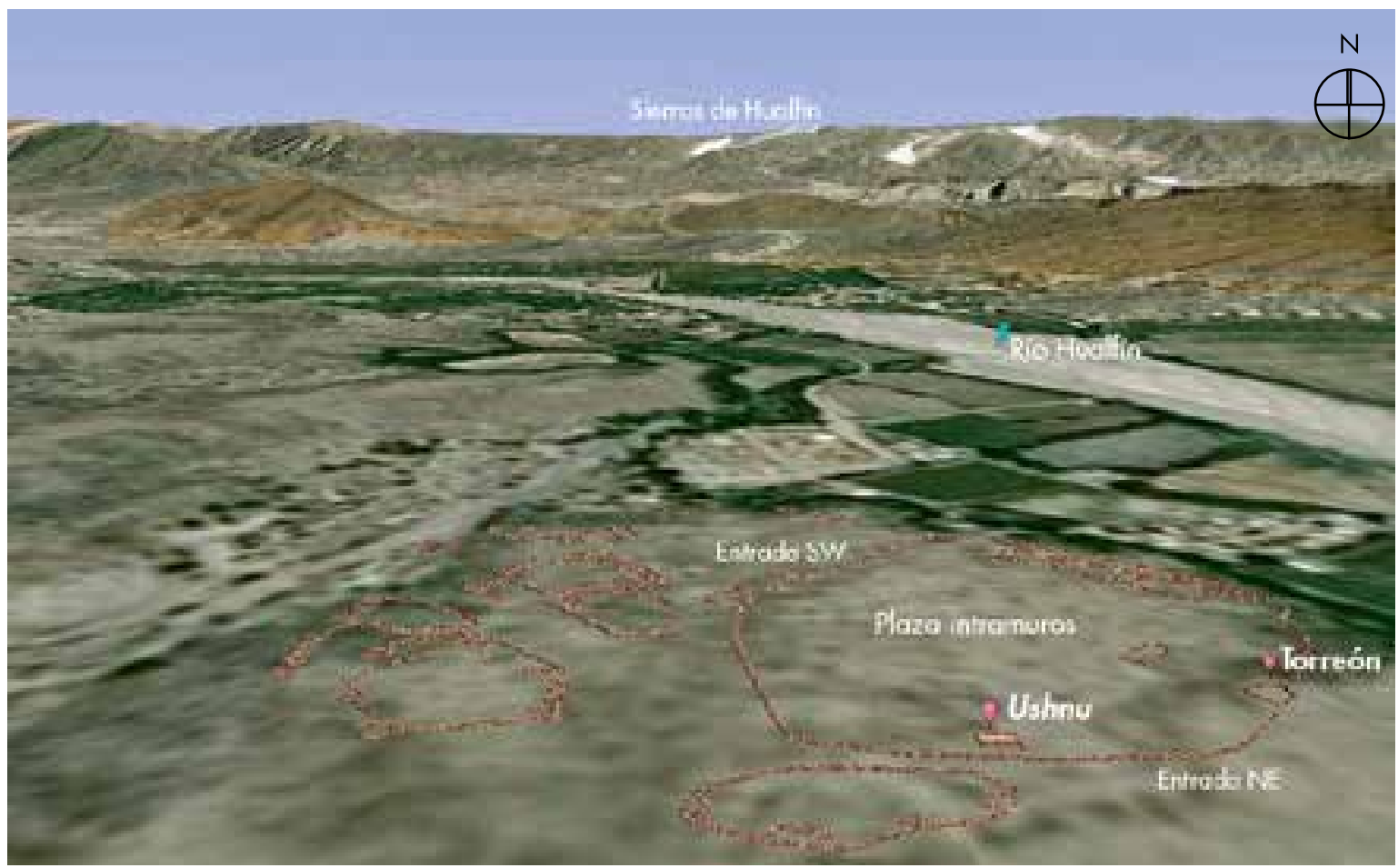

Figura 14. Perspectiva del sitio indicando las entradas a la plaza (NE-SW), que estarían conectadas con el camino inkaico. Figure 14. Outline of the site indicating the entrances to the plaza (NE-SW), which connected with the Inka Road. 
Cada uno de los espacios secundarios que rodean la plaza tiene características diferentes, de acuerdo principalmente a su funcionalidad. Un ejemplo de ello, es quizás el originalmente identificado como un acllabuasi (Raffino 1982) que se encuentra hacia el $\mathrm{N}$ de la plaza. Por sus características, esta estructura podría tratarse de una organización de tipo lineal, conformada por una serie de espacios interrelacionados entre sí, o bien enlazados por otro espacio lineal independiente y distinto (Ching 1993). Aquí vemos que esta estructura está compuesta por una serie de espacios repetidos que son similares en tamaño, forma y función. En este caso, se tratarían de habitaciones para las acllas o mujeres especializadas en actividades productivas, particularmente los textiles y la fabricación de chicha, y que estaban obligadas a prestar servicios laborales al Estado. Estos edificios -que fueron erróneamente interpretados por los cronistas, como Garcilaso de la Vega (1963 [1604]), como conventos cristianos- se encuentran distribuidos en los grandes centros provinciales del Tawantinsuyu. Lamentablemente, para el caso de Hualfín Inka, esta estructura ha sido devastada mayormente por actividades antrópicas, conservándose solo los cimientos de las estructuras que componen este espacio, haciendo imposible su excavación sistemática (Lynch 2010).

Tomando nuevamente a Ching (1993), uno de los puntos a tener en cuenta es la aproximación al sitio desde distintas vías de acceso. Podríamos decir que se accede a él de una forma más bien frontal, es decir, el acceso conduce directamente a la entrada del sitio a lo largo de un recorrido directo y axial. El objetivo visual que pone fin a la aproximación es nítido, sea toda la fachada del sitio o una entrada situada en la misma. Por otra parte, la entrada al mismo, a un espacio interior o a un campo determinado del espacio exterior, comporta el acto de penetrar a través de un plano vertical que distingue a un espacio de otro y separa el "aquí" del "allî". En términos de localización, la entrada puede estar centrada o descentrada en el plano frontal de un edificio; en este último caso, creará unas condiciones de simetría propias. La situación del acceso, respecto de la forma del espacio adyacente, determinará la disposición del recorrido y el tipo de actividades que ahí se desarrollen. A su vez, la noción de acceso puede reforzarse visualmente haciendo que la apertura sea más baja, ancha y estrecha que lo esperado; practicando un acceso de mucha mayor profundidad y además sinuoso, o articulando la abertura con los elementos decorativos. Para el caso de Hualfín Inka, vemos que uno de los accesos principales al sitio es a través de un espacio en el muro perimetral de la plaza, pudiendo acceder a ella y observar su gran extensión y las estructuras secundarias adosadas. Quizás esta entrada estaría ligada al camino inka o Qhapaq Nan proveniente del norte (fig. 15).

Otro punto a tener en cuenta al momento de analizar el emplazamiento del sitio, sus vías de acceso y su estrategia de visualización, es la forma del espacio de circulación dentro del mismo. Se sabe que los espacios circulatorios constituyen una parte integral de la organización de cualquier edificio y ocupan una cantidad importante del volumen del mismo. Los recorridos de circulación, considerados simplemente como dispositivos de unión, darían lugar a interminables espacios-pasillo. Por lo tanto, la forma y la escala del espacio circulatorio deben ser apropiadas al desplazamiento de la persona, ya sea un paseo, una actividad cotidiana, un descanso, la contemplación del paisaje, etc. Es por ello que la forma de un espacio de circulación varía según estén definidos sus límites; se relacione su forma a la de los espacios que comunica; se articulen su escala, su proporción, su iluminación y sus vistas; las peculiaridades de sus accesos; utilice cambios de nivel mediante escaleras y rampas (Ching 1993).

Por su parte, un espacio circulatorio puede ser cerrado, formando un pasillo que relacione todos los espacios, a los que comunica a través de entradas practicadas en el plano de la pared; abierto por un lado, para suministrar una continuidad visual y espacial con los espacios que une, y abierto por ambos lados, para así convertirse en una prolongación de los espacios que atraviesa.

La anchura y la altura de todo espacio circulatorio deben estar proporcionados a la clase y la cantidad de tráfico que tenga que canalizar. Una vía estrecha y cerrada estimulará la circulación. En ocasiones es conveniente ensanchar un recorrido concreto con objeto de habilitar ámbitos para detenerse, descansar o contemplar el paisaje, igualmente se agrandará al acoplarlo con los espacios que traspasa. Situado en un espacio suficientemente amplio, como es el caso de la plaza principal del sitio, un recorrido puede ser arbitrario, sin forma o definición clara, y siempre subordinado a las actividades que se desarrollen en aquel. Para este caso la plaza funcionaría como un espacio abierto con funciones de conglomerar grandes cantidades de personas en ciertas ocasiones, como eran las fiestas o ceremonias practicadas en ciertas épocas del año.

Otro punto a tener en cuenta es el principio de jerarquía. Una vez más, en palabras de Ching (1993), podemos decir que este implica que en la mayoría -si no en el total- de las composiciones arquitectónicas existen auténticas diferencias entre las formas y los espacios, que en cierto sentido reflejan su grado de importancia y el cometido funcional, formal y simbólico que juegan 

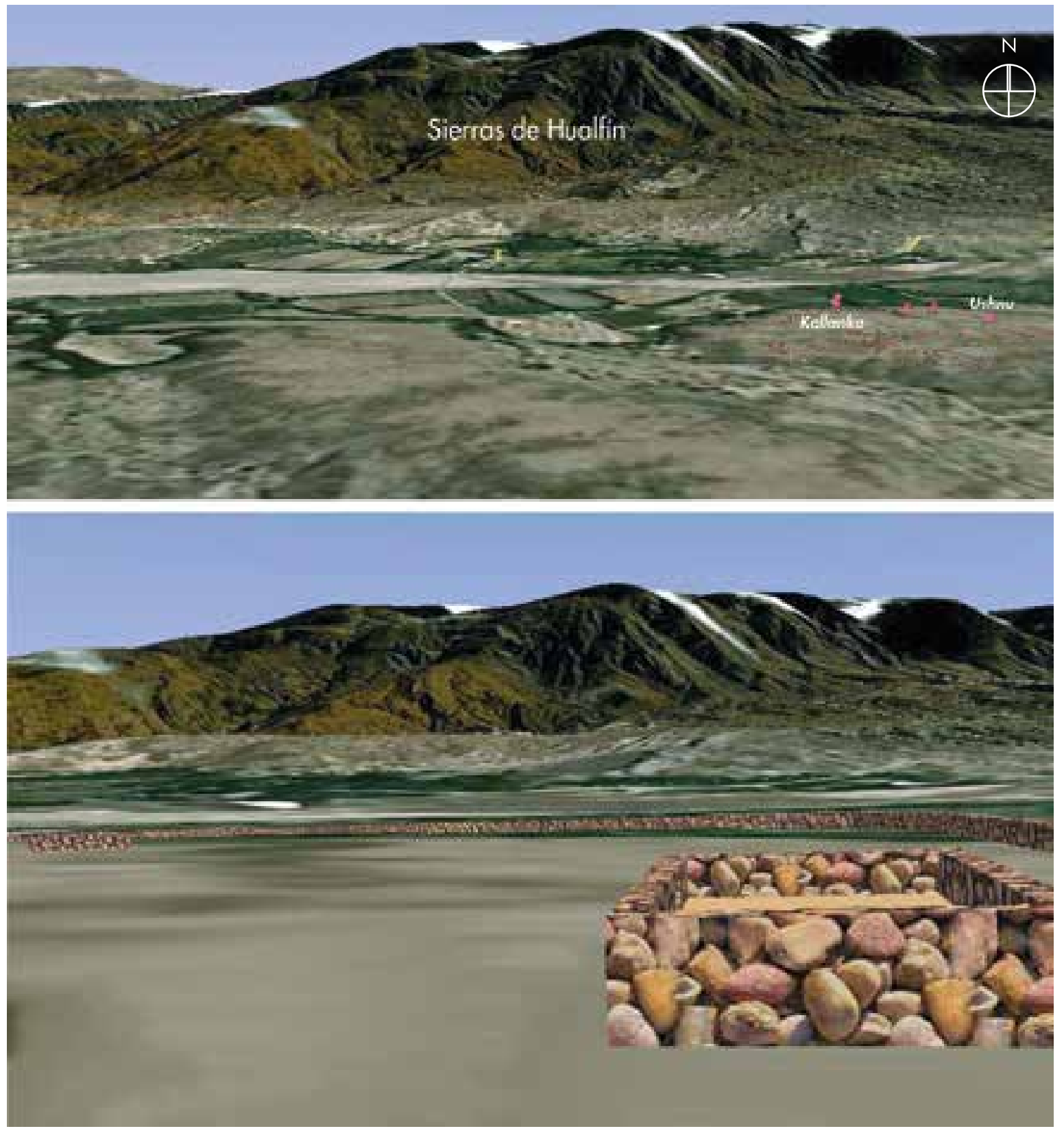

Figura 15. a) Perspectiva de visualización desde el sitio hacia los sitios locales encontrados en la inmediatez del mismo y los principales rasgos naturales del valle; b) Vista panorámica desde el ushnu dentro de la plaza.

Figure 15. a) View from the site looking toward other sites in the immediate area and the surrounding landscape of the valley; b) Panoramic view from the ushnu inside the plaza.

en su organización. El sistema de valores con el que se mide su importancia relativa depende, sin duda, del caso en concreto, de las necesidades y deseos de los usuarios y de las decisiones del diseñador. Los valores empleados pueden ser de carácter individual o colectivo, personal o cultural. En cualquier caso, el modo como se manifiestan estas diferencias funcionales o simbólicas entre los elementos de una edificación es un juicio a la exposición de un orden patente y jerárquico en las formas y espacios que la componen. La articulación de una forma o de un espacio con el propósito de darle importancia o significación debe llevarse a cabo de modo claramente exclusivo y unitario. Esto se puede alcanzar dotándole de una dimensión excepcional, una 
forma única y una localización estratégica. En definitiva, la predominancia de una forma o espacio que es jerárquicamente importante se logra convirtiéndolo en una excepción a la norma, en una anomalía dentro de un modelo que, de no ocurrir así, sería regular. Una forma o un espacio pueden dominar una composición arquitectónica al destacar por su tamaño entre todos los elementos integrantes. Por lo general, este dominio se hace visible por las dimensiones del elemento, aunque puede darse el caso en que, precisamente, un elemento sobresalga por su pequeñez y por una localización claramente indicada. Tal es el caso de la plaza y del ushnu, por el tamaño del primero y la localización estratégica del segundo, produciendo un impacto visual en las personas que llegaban al sitio.

Por último, vemos que la utilización del paisaje y la arquitectura planteada por los inkas al momento de la llegada al valle de Hualfín, trajo cambios en el uso, la reorganización y el significado de los espacios públicos, domésticos y ceremoniales de las poblaciones locales.

Para el caso del sitio Hualfín Inka, teniendo en cuenta la propuesta de Williams (2004), presentó muchas características que lo habrían ubicado en un lugar preponderante dentro de una política de administración indirecta de las poblaciones locales. En este sentido, su gran plaza parece haber servido en un primer momento para reunir a una gran cantidad de personas para que tributaran o participaran en actos de secularización, a través de ceremonias organizadas y dirigidas por la elite local (curacas locales de una posición social importante y vinculados directamente con el Inka) que allí residía, o practicando la redistribución de bienes de consumo con el objetivo de recrear su poder. Elaborados de forma conjunta el ushnu y la organización del espacio crearon una conciencia colectiva uniforme, al tomar ideas que existían en los territorios conquistados y usarlas para legitimar el dominio ideológico del Imperio.

Finalmente, vemos para el caso de Hualfín Inka que su construcción constituyó un poderoso mecanismo de dominación ideológica y cultural en un primer momento, de acuerdo con su arquitectura con características de grandes centros administrativos, un tipo de política indirecta sobre las poblaciones locales del valle de Hualfín y, por ende, una relación de alianza o de reciprocidad dentro de la gran variabilidad de las estrategias políticas estatales planteadas para las zonas más meridionales del Tawantinsuyu.

RECONOCIMIENTOS En primer lugar al Museo y la Facultad de Ciencias Naturales de la ciudad de La Plata por la formación y el espacio brindados, que han permitido la realización de tareas varias de investigación, algunas de las cuales se detallan en el presente trabajo; a quienes han colaborado en las tareas de campo, de laboratorio; a la localidad de Hualfín por permitir la concreción de las mismas y en particular a las entidades que permiten la divulgación de tales labores.

\section{NOTAS}

1 Acordamos utilizar el término "imperio" para el inkario por considerarlo un Estado expansivo, en el cual el núcleo social central ejerció un control sobre las distintas sociedades asentadas en el territorio, estableciendo como norma de integración/identificación una serie de condicionamientos variables de acuerdo con las distintas realidades regionales.

2 Laboratorio NSF de AMS, Edificio de Física, Universidad de Arizona, Estados Unidos.

\section{REFERENCIAS}

AcuTo, F., 1999. Paisaje y dominación: La constitución del espacio social en el imperio Inka. En Sed non satiata. Teoría social en la arqueología Latinoamericana Contemporánea, A. Zaranquin \& F. Acuto, Eds., pp. 33-75. Buenos Aires: Ediciones del Tridente.

AlBornoz, C., 1967 [1582]. La instrucción para descubrir todas las guacas del Pirú y sus camayos y haziendas. Journal de la Société des Américanistes 56: 9-39.

Aschero, C., 1975 Ms. Ensayo para una clasificación morfológica de artefactos líticos aplicada a estudios tipológicos comparativos. Informe al CONICET.

— 1983 Ms. Ensayo para una clasificación morfológica de artefactos líticos. Apéndices A y B. Apunte de la Cátedra de Ergología y Etnología, Buenos Aires.

Balesta, B. \& F. Wynveldt, 2010. La Loma de Ichanga: Visibilidad, defensibilidad y abandono en el valle de Hualfín (Depto. de Belén, Prov. de Catamarca, Argentina). Revista Española de Antropología Americana 40 (1): 53-71, Madrid.

Borgstede, G. \& J. R. Mathieu, 2007. Defensibility and Settlement Patterns in the Guatemalan Maya Highlands. Latin American Antiquity 18 (2): 191-211.

Bruch, C., 1904. Descripción de algunos sepulcros calchaquíes. Resultado de las excavaciones efectuadas en Hualfín (Provincia de Catamarca). Revista del Museo de La Plata, Tomo XI. Buenos Aires.

Caminos, R., 1979. Sierras pampeanas noroccidentales, Salta, Tucumán, Catamarca, La Rioja y San Juan. Geología Regional Argentina. Academia Nacional de Ciencias: 225-282, Córdoba.

Ceruti, C., 1997. Arqueología de alta montaña, Mendoza: Ed. Milor.

Ching, F., 1993. Arquitectura: forma, espacio y orden. México, D. F.: Ediciones Gustavo Gili.

Criado BoAdo, F., 1999. Del terreno al espacio: planteamientos y perspectivas para la arqueología del paisaje. CAPA 6. Santiago: Laboratorio de Arqueoloxía e Formas Culturais.

D'Altroy, T., 1987. Transitions in power: Centralization of Wanca Political Organization under Inka rule. Ethnobistory 34: 78-102.

D'Altroy, T.; A. M. Lorandi \& V. Williams, 1998. Ceramic Production and use in the Inka Political Economy. En Andean Ceramic Technology, Organization and Approaches, I. Shimada, Ed., pp. 283-312. Philadelphia: Museum of Archaeology and Anthropology, University of Pennsylvania.

Garcilaso de la Vega, I., 1963 [1604]. Comentarios reales de los incas (primera parte). Biblioteca de Autores Españoles, Vol. 133, Madrid.

GiovannetTi, M., 2009. Articulación entre el sistema agrícola, redes de irrigación y áreas de molienda como medida del grado de ocupación Inka en El Shincal y Los Colorados (Prov. de Catamarca). Tesis de doctorado inédita. FCNyM, UNLP.

Giovannetti, M.; G. Cochero, P. Espósito \& J. Spina, 2010. Excavación y análisis de un mortero múltiple a través de la diversidad de 
su registro y su relación con la evidencia cerámica. En Actas del XVII Congreso Nacional de Arqueología Argentina, pp. 163-168, Mendoza.

González Bonorino, F., 1950. Algunos problemas geológicos de las Sierras Pampeanas. Revista de la Asociación Geológica Argentina 5 (3): 81-110, Buenos Aires.

GuAmán POMA DE AYALA, F., 1980 [1615]. El primer nueva corónica y buen gobierno. México, D. F.: Editorial Siglo Veintiuno.

HERnÁNDEZ PrínciPe, R., 1923 [1641-1642]. Mitología andina. Idolatría en Recuay. Revista Inca 1 (1): 24-78, Lima.

Hyslop, J., 1984. The Inka road System. New York / San Francisco: Academic Press.

- 1990. Inka Settlement planning. Austin: University of Texas Press. InOMATA, T. \& L. S. COBEN, 2006. Archaeology of Performance: Theaters of Power and Community. Walnut Creek, CA: Altamira Press.

LYNCH, J., 2010. La construcción del paisaje y la organización del espacio en el sector norte del valle de Hualfín, Provincia de Catamarca, Tesis doctoral inédita. Facultad de Ciencias Naturales y Museo. Universidad Nacional de La Plata, Argentina.

Lynch, J.; V. Lynch \& R. PÁEZ, 2006. Análisis preliminar de los materiales recuperados del sitio arqueológico Hualfín Inka (Departamento Belén, Catamarca). En Actas del III Congreso de Historia de Catamarca, Tomo I, pp. 197-203. San Fernando del Valle de Catamarca: Editorial Científica Universitaria.

LynCH, J.; V. Lynch, V. LISSA \& R. PÁEz, 2007. Primeros avances de los materiales recuperados del sitio Hualfín Inka, Depto. Belén, Provincia de Catamarca. Revista Pacarina, Número especial, Tomo II: 525-532. San Salvador de Jujuy: Ed. Universidad Nacional de Jujuy.

LYNCH, J. \& C. PÁEz, 2009. Características de la relación local-estatal en el sitio Hualfín Inka (Depto. Belén, Provincia de Catamarca, Argentina). Inferencias a partir del análisis cerámico. Revista Arqueoweb (Revista sobre arqueología en internet), Número 13-2011 [online] pp. 55-73, <http://pendientedemigracion.ucm. es/info/arqueoweb/numero-13.html\#13>, ISSN: 1139-9201, Universidad Complutense de Madrid, España.

Lynch, J.; M. GiovannetTI \& M. C. PÁEz, 2010. Ushnus de las provincias del sur del Tawantinsuyu. Comparación entre las estructuras de los sitios El Shincal y Hualfín Inka en el centro de Catamarca (Argentina). Revista de Arqueología Americana 28: 49-81.

LyNCH, J. \& V. LynCH, 2010. Análisis de materiales líticos y aprovisionamiento de materias primas del sitio arqueológico Hualfín Inka (Provincia de Catamarca). En Actas del XVII Congreso Nacional de Arqueología Argentina, pp. 1685-1690, Mendoza.

Matos, R., 1986. El ushnu de Pumpu. Cuicuilco 18: 45-61, México, D. F.

— 1994. Pumpu: Centro administrativo inca de la Puna de Junín. Lima: Editorial Horizonte.

Meddens, F., 1997. Function and meaning of the ushnu in Late Horizon Perú. Tawantinsuyu 3: 4-14, Camberra-La Plata.
Moore, J., 1996. The archaeology of plazas and the proxemics of ritual: three Andean traditions. American Anthropologist, New Series 98 (4): 789-802.

Nielsen, A., 2006. Plazas para los antepasados: Descentralización y poder corporativo en las formaciones políticas preincaicas de los Andes Circumpuneños. Estudios Atacameños 31: 63-89.

Nielsen, A. \& L. Boschi, 2007. Celebrando con los Antepasados Arqueología del espacio público en Los Amarillos, Quebrada de Humahuaca, Jujuy, Argentina. Buenos Aires: Mallku Ediciones.

Nielsen, A. \& W. Walkers, 1999. Conquista ritual y dominación política en el Tawantinsuyu: el caso de Los Amarillos (Jujuy, Argentina). En Sed non satiata. Teoría social en la arqueología Latinoamericana Contemporánea, A. Zaranquin \& F. Acuto, Eds., pp. 153-169. Buenos Aires: Ediciones del Tridente.

Pino Matos, J., 2004. El ushnu inka y la organización del espacio en los principales tampus de los wamani de la sierra central del Chinchaysuyu. Chungara 36 (2): 303-311.

RafFino, R., 1981. Los inkas del Kollasuyu. Origen, naturaleza y transfiguraciones de la ocupación Inka en los Andes Meridionales. La Plata: Ed. Ramos Americana.

— 2004. El Shincal de Quimivil. Catamarca: Ed. Sarquís

Raffino, R.; R. Alvis, L. Baldini, D. Olivera \& G. Raviña, 1982. Hualfín-El Shincal-Watungasta. Tres casos de urbanización inka en el N. O. argentino. En Actas del IX Congreso Nacional de Arqueología Chilena, pp. 470-497. La Serena: Museo Arqueológico de La Serena.

Raffino, R.; D. Gobbo, R. Vázquez, A. Capparelli, V. Montes, D. Iturriza, C. Deschamps \& M. Mannasero, 1997. El ushnu de El Shincal de Quimivil. Tawantinsuyu 3: 22-39, Camberra-La Plata.

REINHARD, J., 1985. Sacred Mountains: an Ethnnoarchaeological study of High Andean Ruins. Mountain Research and Development 5 (4): 299-317.

SÁnchez, R., 2004. El Tawantinsuyu en Aconcagua (Chile Central). Chungara 36 (2): 325-336.

SCHobinger, J., 1966. Investigaciones arqueológicas en la Sierra de Famatina (Provincia de La Rioja). Anales de Arqueología y Etnología 21: 139-196, Mendoza.

— 1971. Arqueología del Valle de Uspallata, Provincia de Mendoza (Sinopsis preliminar). Relaciones de la Sociedad Argentina de Antropología, NS, tomo V (2): 71-84, Buenos Aires.

STEHBERG, R., 1995. Instalaciones incaicas en el norte y centro semiárido de Chile. Santiago: Dirección Bibliotecas, Archivos y Museos, Centro de Investigación D. Barros Arana.

Tilley, C., 1994. A Phenomenology of Landscape. Places, Paths and Monuments. Oxford: Berg.

VITRY, C., 2000. Aportes para el estudio de caminos incaicos. Tramo Morohuas-Incahuasi. Salta: Ed. Gofica.

Williams, V., 2004. Poder estatal y cultura material en el Kollasuyu. Boletín de Arquelogía PUCP 8: 209-245, Lima.

Zuidema, R., 1980. El ushnu. Revista de la Universidad Complutense 117: 317-362, Madrid. 